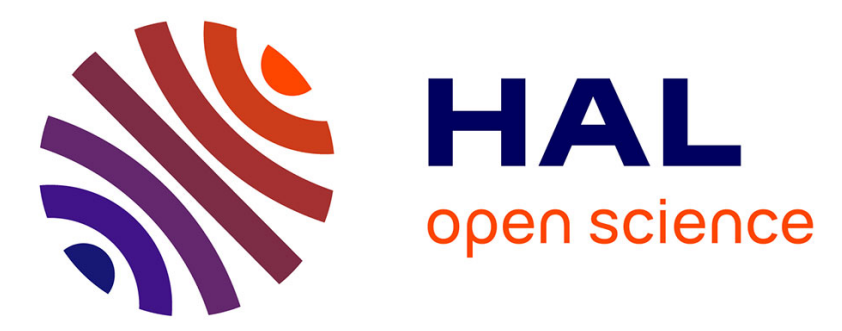

\title{
Estimation of the orbital angular momentum order using a vector antenna in the presence of noise
}

Cheikh Dieylar Diallo, Duy Kevin Nguyen, Alexandre Chabory, Nicolas Capet

\section{To cite this version:}

Cheikh Dieylar Diallo, Duy Kevin Nguyen, Alexandre Chabory, Nicolas Capet. Estimation of the orbital angular momentum order using a vector antenna in the presence of noise. EuCAP 2014, 8th European Conference on Antennas and Propagation, Apr 2014, The Hague, Netherlands. pp.3248 3252, 10.1109/EuCAP.2014.6902521 . hal-01108946

\section{HAL Id: hal-01108946 \\ https://hal-enac.archives-ouvertes.fr/hal-01108946}

Submitted on 23 Jan 2015

HAL is a multi-disciplinary open access archive for the deposit and dissemination of scientific research documents, whether they are published or not. The documents may come from teaching and research institutions in France or abroad, or from public or private research centers.
L'archive ouverte pluridisciplinaire HAL, est destinée au dépôt et à la diffusion de documents scientifiques de niveau recherche, publiés ou non, émanant des établissements d'enseignement et de recherche français ou étrangers, des laboratoires publics ou privés. 


\title{
Estimation of the Orbital Angular Momentum Order Using a Vector Antenna in the Presence of Noise
}

\author{
Cheikh Dieylar DIALLO ${ }^{1,2}$, Duy Kevin NGUYEN ${ }^{1,4}$, Alexandre CHABORY ${ }^{2,3}$, Nicolas CAPET ${ }^{1}$ \\ ${ }^{1}$ CNES, antenna department, 18 avenue Edouard Belin, Toulouse, France \\ ${ }^{2}$ ENAC, TELECOM-EMA, F-31055 Toulouse, France \\ ${ }^{3}$ Université de Toulouse, F-31400 Toulouse, France \\ ${ }^{4}$ Université de Toulouse: UPS, INPT, LAPLACE, Toulouse, France and CNRS: LAPLACE, Toulouse, France
}

\begin{abstract}
The use of the orbital angular momentum (OAM) may be of interest in the radio-frequency spectrum notably to multiple the number of communication channels. Another way to use this property is to transmit data through the OAM state (Qbit) of the transmitted wave. In a noiseless environment, the local estimation of the OAM order can be realized by means of a vector antenna that measures the 6 components of the electromagnetic field. In this article, we study the sensitivity to noise of this estimation. The OAM wave is generated via Laguerre-Gauss beams. For several mode orders, signal-to-noise ratio and distances to the transmitter, we perform a statistical analysis from which we obtain a probability of error in the order estimation. We demonstrate that the local estimation of the OAM order is very sensitive to noise, especially in the far-field zone.
\end{abstract}

Index Terms-OAM, orbital angular momentum, GaussLaguerre, noise study, vector antenna, local estimation, local detection, rotation phase fronts.

\section{INTRODUCTION}

From electromagnetic literature, it is well-known that a wave carries energy and momentum. The momentum may have linear and angular components [1]. The angular momentum can be decomposed into a spin angular momentum (SAM) and an orbital angular momentum (OAM).

In optics, the SAM has been observed since the thirties [2]. Conversely, it is only in 1992 [3] that a practical use of the OAM has been realized. The SAM corresponds to polarization. Thus, it only has two independent states, while, theoretically, there exists an infinite number of states for the OAM.

In 2007 [4], the first radio OAM simulations were performed and 5 years later [5] the first radio OAM transmission was experimented. In recent papers, the use of the different OAM states for increasing the capacity of wireless communications has been controversially discussed, notably via a MIMO analysis [6]. The purpose of this paper is not to answer this question but to determine whether the OAM order of a wave can be measured locally.

As demonstrated in [7], it is possible to estimate unambiguously and locally the OAM order of an electromagnetic wave, knowing the beam axis and the 6 components of the electromagnetic fields. However, this result has been presented in the absence of noise, which is not realistic for any practical application.

In this article, we study locally the estimation of the OAM order in presence of noise using a single or an array of vector antennas (VA). This antenna measures at one or several points the 6 components of the electromagnetic fields, with complete amplitude and phase information.

\section{ORBITAL ANGULAR MOMENTUM}

\section{A. Definition and quantification}

In the frequency domain, the angular momentum density associated with an electromagnetic field can be written as

$$
\boldsymbol{J}=\varepsilon_{0} \int \boldsymbol{r} \times \operatorname{Re}(\boldsymbol{E} \times \boldsymbol{H}) d V,
$$

with $\boldsymbol{r}$ the position vector, $\boldsymbol{E}$ and $\boldsymbol{H}$ the electric and magnetic fields, respectively. As explained in [8], the angular momentum can be decomposed into two components. The intrinsic component, called the Spin Angular Momentum (SAM), corresponds to the polarization of the wave, and is independent of the coordinate system. On the contrary, the extrinsic component depends on the coordinate system, and is referred to as the Orbital Angular Momentum (OAM).

With the previous decomposition, the angular momentum order $j$ is the sum of the SAM and OAM orders denoted $s$ and $l$, respectively. We obtain

$$
j=s+l=\frac{\omega J_{z}}{\frac{\varepsilon_{0}}{2}\left(\|\boldsymbol{E}\|^{2}+\|\boldsymbol{H}\|^{2}\right)},
$$

where $J_{z}$ is the $z$-component of $\boldsymbol{J}$, i.e.

$$
J_{z}=\varepsilon_{0} \int \operatorname{Re}\left\{x\left(E_{z} B_{x}-E_{x} B_{z}\right)-y\left(E_{y} B_{z}-E_{z} B_{y}\right)\right\} d V .
$$

In this paper, we only use linearly polarized waves for which $s=0$, thus $j=l$. Besides, we only consider pure OAM modes, for which $l$ is an integer.

\section{B. Receiver topology for a local estimation of the OAM order}

The system considered in this article is presented in Fig. 2. It consists of an OAM transmitter and a local receiver capable of estimating the OAM order. From (3), to estimate $l$ we need to know $x$ and $y$, i.e. the position of the receiver with respect to the transmitter $z$-axis. We also need to measure the 6 components of the electromagnetic fields, with complete amplitude and phase information. Thus, an ideal receiver would be a vector antenna (VA) consisting of 6 co-located elements: three short electric dipoles and three short magnetic 
dipoles [9]. In the following, the vector antenna is assumed to be ideal: composed of ideal electric and magnetic dipoles without coupling. In this article, we consider different topologies of VA arrays that comprise between 1 and 9 elements, and are of size from $0.1 \lambda$ to $\lambda$, where $\lambda$ is the wavelength as shown in Fig. 1.

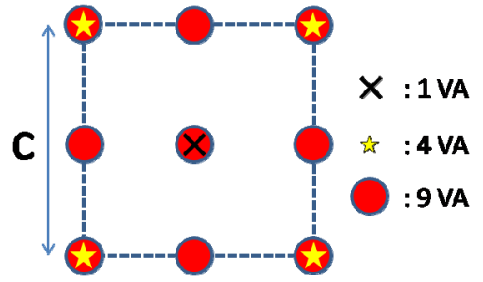

Fig. 1. Topologies of receivers with $c=0.1 \lambda$ or $c=\lambda$.

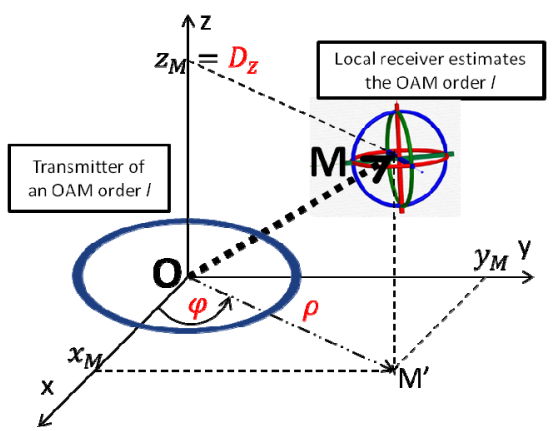

Fig. 2. OAM transmitting and receiving simulation system.

\section{ANALYTIC FORMULATION OF A WAVE CARRYING AN OAM}

From optics literature [3], we know that paraxial LaguerreGauss beams carry a pure OAM order. Furthermore, their analytic expressions are known and the azimuthal phase dependence is explicit. This makes the Laguerre-Gauss modes very useful and simple for modelling and studying an OAM carrying wave.

\section{A. Laguerre-Gauss beams}

The Laguerre-Gauss beams are solutions of the paraxial propagation equation. In cylindrical coordinates $(\rho, \varphi, z)$, their amplitude is given by

$$
\begin{aligned}
& U_{n}^{l}(\rho, \varphi, z)=\sqrt{\frac{2 n !}{\pi[(|l|+n) !]^{3}}} \frac{1}{W(z)} \exp \left(-\left[\frac{\rho}{W(z)}\right]^{2}\right) \\
& {\left[\sqrt{2} \frac{\rho}{W(z)}\right]^{|l|} L_{n}^{|l|}\left(2 \frac{\rho^{2}}{W(z)^{2}}\right) \exp \left(-i\left[\frac{k \rho^{2}}{2 R(z)}\right]\right)} \\
& \exp (i l \varphi) \exp \left(i\left[(2 n+|l|+1) \arctan \left(\frac{z}{z_{0}}\right)-k z\right]\right),
\end{aligned}
$$

where $k$ is the wave number, $L_{n}^{|l|}$ are the Laguerre polynomials, and $(l, n)$ are integers that define the orbital and radial mode orders, respectively. The expressions of $W(z)$ and $R(z)$ are given by

$$
W(z)=W_{0} \sqrt{\left(1+\left(\frac{z}{z_{0}}\right)^{2}\right)},
$$

and

$$
R(z)=\frac{z^{2}+z_{0}^{2}}{z}
$$

with $W_{0}$ the beam waist size and $z_{0}$ the Rayleigh distance. As presented in Fig. 3, $W(z)$ represents the radius of the beam envelope, at which the Gaussian function has dropped of 1/e from its axial value. Furthermore, $R(z)$ describes the curvature radius of the wavefront on the $z$ axis.

Note the azimuthal phase dependency, $\exp (i l \varphi)$, in the analytic expression of the Laguerre-Gauss complex amplitude.

The Laguerre-Gauss beams depend on the paraxial approximation, which assumes that the field is weakly diverging with propagation. This approximation is known to be valid when the field is confined in a cone of axis $z$ and of angle $22^{\circ}$. This corresponds to $W_{0}>0,7 \lambda$.

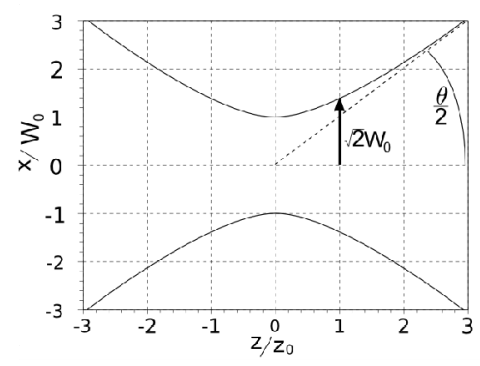

(a)

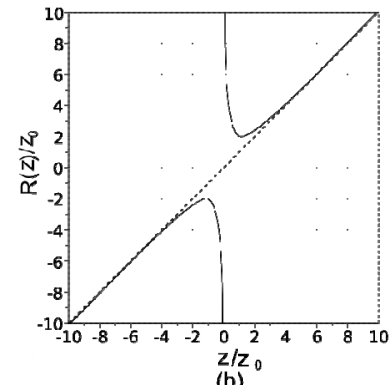

(b)
Fig. 3 (a) Shape of a Gaussian beam in the plane $(x O z)$. (b) Radius of curvature of the wave front on the $z$-axis.

In the following, only pure orbital modes, defined by $n=0$, are used. From the scalar solution (4), one can derive a vectorial formulation for the Laguerre-Gauss beams [10]. This yields

$$
\begin{aligned}
& \boldsymbol{E}_{L G}=E_{0}^{l}=U_{0}^{l} \boldsymbol{e}_{x}-\frac{i}{k} \frac{\partial U_{0}^{l}}{\partial x} \boldsymbol{e}_{z}, \\
& \boldsymbol{H}_{L G}=\boldsymbol{H}_{0}^{l}=\frac{1}{Z_{0}}\left(U_{0}^{l} \boldsymbol{e}_{y}-\frac{i}{k} \frac{\partial U_{0}^{l}}{\partial y} \boldsymbol{e}_{z}\right),
\end{aligned}
$$

where $Z_{0}$ is the free space wave impedance.

\section{B. Illustrations and validations}

In [7], a system study has been presented for OAM modes generated by a circular array antenna. It has been found that from the 6 components of the electromagnetic fields, with complete phase information, it is possible to estimate the OAM order by local measurements at a single point, assuming noiseless conditions and a known beam axis. We here perform a similar analysis but with the Laguerre-Gauss formulation.

In Fig. 4, we observe the spiral phase variations in a transverse plane for two different values of $l$. This result is one of the main properties of a wave with a non-zero OAM order. Besides, it is consistent with [7]. This result also shows that the phase variation gets faster when $|l|$ is higher. 

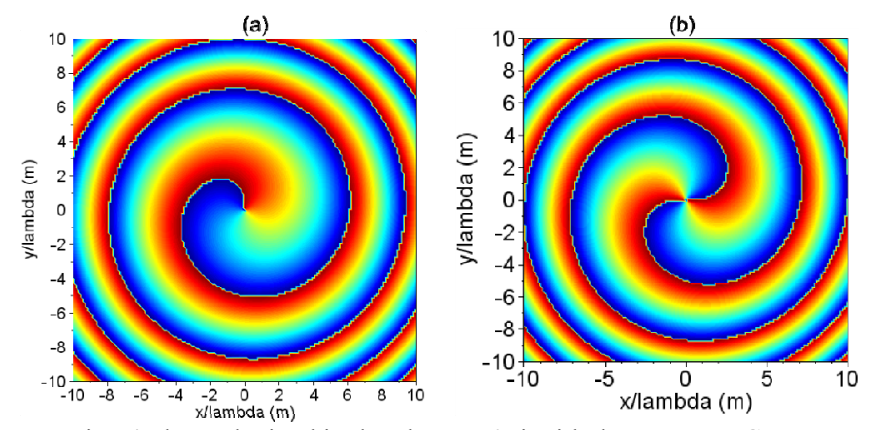

Fig. 4 Phase obtained in the plane $z=25 \lambda$ with the Laguerre-Gauss formulation for $W_{0}=2 \lambda$, and for either $l=1$ (a) or $l=2$ (b).

In Table I, we compare the influence of the transmitter dimension, characterized by $\mathrm{W}_{0}$, on the maximum gain value and position. As in [7], when the antenna size becomes smaller, the angle of the maximum gain increases while the gain decreases.

TABLE I. INFLUENCE OF THE TRANSMITTER SIZE ON THE MAXIMUM GAIN VALUE AND POSITION FOR $L=1$

\begin{tabular}{|c|c|c|}
\hline$W_{0}(\lambda)$ & $\Theta\left({ }^{\circ}\right)$ & $G^{\max }(\mathrm{dB})$ \\
\hline 1.0 & 13.4 & 15.0 \\
\hline 1.5 & 9.1 & 18.0 \\
\hline 2.0 & 7.3 & 19.8 \\
\hline
\end{tabular}

The previous simulations have shown the properties (phase and radiation pattern) of a Gauss-Laguerre beam with a nonzero OAM order. We now estimate whether an array of vector antennas can be used to locally measure the OAM order. In Fig. 5 , in the plane $z=25 \lambda$, we estimate $l$ with (2) for a beam such that $W_{0}=2 \lambda$ and $l=2$ as a function of the distance to the $z$ axis. The results show that all receivers achieve to estimate locally the OAM order except in the null beam axis. Moreover, with only one element, we obtain the best estimation. With 4 elements, the local estimation improves as the size of the receiver decreases.

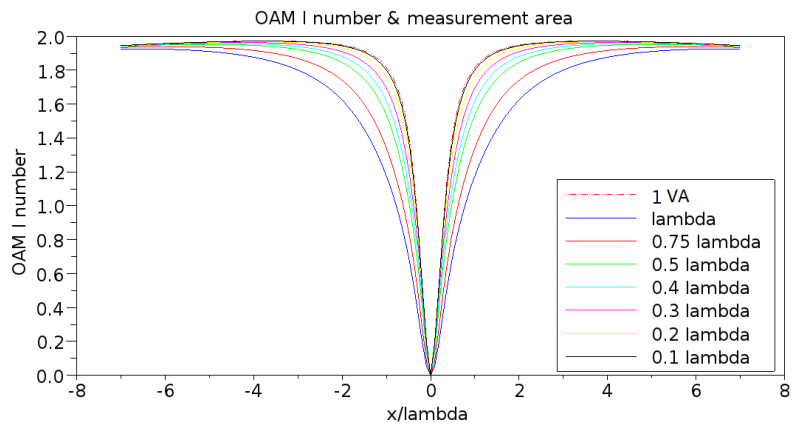

Fig. 5 Estimation of the OAM order for $W_{0}=2 \lambda$ and $l=2$ in the plane $z=25 \lambda$ with 8 receiver topologies: $1 \mathrm{VA}$ and arrays of $4 \mathrm{VA}$ with $c$ from $0.1 \lambda$ to $\lambda$.

In Table II, we place the receiver at the greatest beam intensity, which location, $\rho_{\max }(z)$ can be analytically calculated from the derivation of (4)

$$
\rho_{\max }(z)=W(z) \sqrt{|l| / 2} \text {. }
$$

TABLE II. DEVIATION $\left|l_{\text {estimated }}-l\right| 10^{-2}$ OF THE LOCALLY ESTIMATED OAM ORDER FOR $l=2, z=50 \lambda$.

\begin{tabular}{|c|c|c|c|c|}
\hline$N_{X Y}{ }^{2}$ & \multicolumn{2}{|c|}{$W_{0}=\lambda$} & \multicolumn{2}{c|}{$W_{0}=2 \lambda$} \\
\hline $1^{2}$ & 4.03 & $\mathbf{4 . 0 3}$ & 2.57 & $\mathbf{2 . 5 2}$ \\
\hline $2^{2}$ & 5.32 & $\mathbf{5 . 3 2}$ & 2.63 & $\mathbf{2 . 5 2}$ \\
\hline $10^{2}$ & 4.56 & $\mathbf{4 . 5 6}$ & 2.58 & $\mathbf{2 . 5 2}$ \\
\hline
\end{tabular}

Even if the error is higher than the one obtained with a circular array [7], this result confirms that the OAM order can be estimated locally in a noiseless configuration.

\section{LOCAL ESTIMATION OF THE OAM WITH NOISE}

To estimate the interest of OAM in RF transmissions, we propose to test the estimation of the OAM order with vectorantennas in presence of noise.

\section{A. Noise definition}

To test the sensitivity of the OAM order local estimation to noise, we add independent Gaussian noises to the real and imaginary parts of the 6 components of the electromagnetic field $\left(E_{x}, E_{y}, E_{z}, H_{x}, H_{y}, H_{z}\right)$, which are generated from Laguerre-Gauss modes. Then, we estimate the OAM order using (2). The total electromagnetic fields are given by

$$
\begin{aligned}
& \boldsymbol{E}_{\text {total }}=\boldsymbol{E}_{L G}+\boldsymbol{E}_{N}, \\
& \boldsymbol{H}_{\text {total }}=\boldsymbol{H}_{L G}+\boldsymbol{H}_{N},
\end{aligned}
$$

where $\boldsymbol{E}_{L G}, \boldsymbol{H}_{L G}$ are the ideal fields coming from LaguerreGauss formulations, as defined in (7) and (8), and $\boldsymbol{E}_{N}, \boldsymbol{H}_{N}$ are the noise vectors. Finally, the noise components can be expressed as

$$
\begin{array}{ll}
E_{N x}=\left(N_{E x}^{\prime}+i N_{E x}^{\prime \prime}\right) & H_{N x}=\left(N_{H x}^{\prime}+i N_{H x}^{\prime \prime}\right) \\
E_{N y}=\left(N_{E y}^{\prime}+i N_{E y}^{\prime \prime}\right) \text { and } & H_{N y}=\left(N_{H y}^{\prime}+i N_{H y}^{\prime \prime}\right), \\
E_{N x}=\left(N_{E z}^{\prime}+i N_{E z}^{\prime \prime}\right) & H_{N x}=\left(N_{H z}^{\prime}+i N_{H z}^{\prime \prime}\right)
\end{array}
$$

where $\left\{N_{E i}^{\prime}, N_{E i}^{\prime \prime}, N_{H i}^{\prime}, N_{H i}^{\prime \prime}\right\}_{i \in\{x, y, z}$ are independent zero-mean normal distributions with standard deviations $\sigma_{E}$ and $\sigma_{H}$, for the electric and magnetic fields, respectively. The noise standard deviations have to be determined. We choose them from a signal-to-noise ratio (SNR) defined by

$$
S N R=\frac{\left\|\boldsymbol{E}_{\max }(z)\right\|^{2}}{\sigma_{E}(z)^{2}},
$$

where $\left|\boldsymbol{E}_{\max }(z)\right|$ is the maximum value of the field for a fixed value of $z$. Therefore, by setting a value to the SNR, we deduce the noise power for any value of $z$

$$
\sigma_{E}(z)^{2}=\frac{\left\|\boldsymbol{E}_{\max }(z)\right\|^{2}}{S N R},
$$

For the magnetic field, we choose $\sigma_{H}=\sigma_{E} / Z_{0}$. In simulations, the noise power will be chosen from realistic values of the SNR in telecommunications, i.e. between $10 \mathrm{~dB}$ and $30 \mathrm{~dB}$. 


\section{B. Results in the far-field zone}

From the developed tool, the OAM order is estimated locally in the far-field zone at $z=10 z_{0}$ for $W_{0}=2 \lambda, l=2$, and a SNR of $30 \mathrm{~dB}$. At each point of the simulation, one sample of the field noise $\boldsymbol{E}_{N}, \boldsymbol{H}_{N}$ is considered. The locally estimated OAM order as a function of the distance to the $z$ axis is shown in Fig. 6 . Regardless of the receiver topology, the estimation is very sensitive to noise even around the highest radiation intensity.

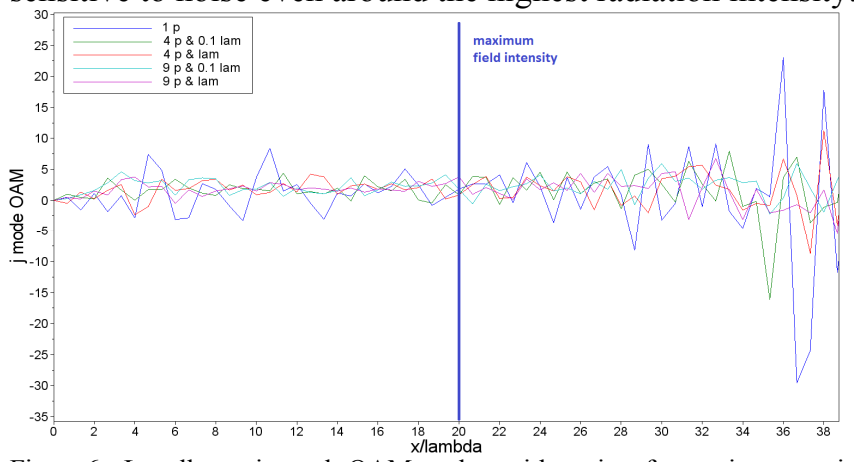

Fig. 6. Locally estimated OAM order with noise for various receiver topologies with $W_{0}=2 \lambda, l=2, z=10 z_{0}, S N R=30 \mathrm{~dB}$.

It appears that we cannot deduce directly the OAM order as in the noiseless case, even around the highest radiation intensity. Besides, the accuracy of the estimation decreases when the distance to the beam axis increases.

\section{Results at shorter distances}

To analyse the sensitivity to noise at shorter distances, we keep the same parameters except the distance $z$, which here is half of the Rayleigh distance $0.5 z_{0}$. In Fig. 7, we observe that the OAM order can be estimated, especially around the highest beam intensity, between $0.86 \lambda$ and $2.8 \lambda$ from the beam axis.

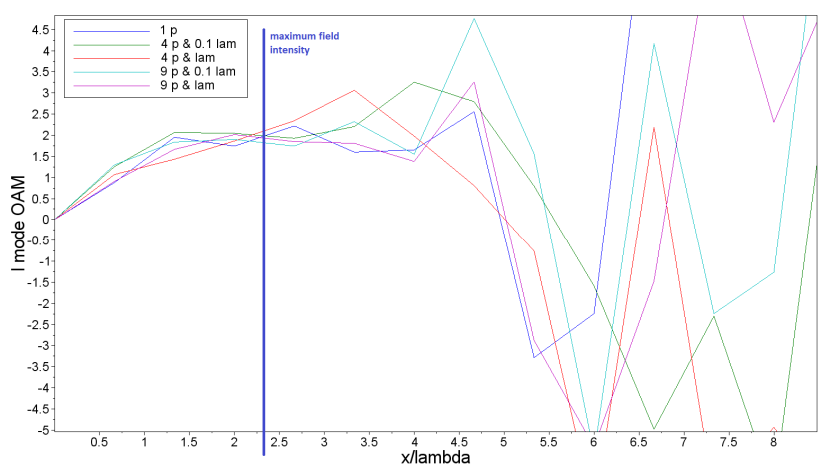

Fig. 7. Locally estimated OAM order with noise for 5 receiver topologies with $W_{0}=2 \lambda, l=2, D_{z}=0.5 z_{0}, S N R=30 \mathrm{~dB}$.

\section{STATISTICAL ANALYSIS}

As noted earlier, the local estimation of the OAM order is very sensitive to noise, especially in far-field zone. For one random sample of the noise, this order does not appear to be measurable directly, but increasing the number of elements in the VA array improves the results. That leads to consider a statistical approach for studying the local estimation of the OAM order with noise.

\section{A. Principle}

The noise is stochastic. Therefore, only a statistical analysis can fully determine its influence on the estimation.

We perform Monte-Carlo simulations to obtain the statistical parameters associated with the local estimation of the OAM order. We use 5 different topologies of receiver, placed at the location of the greatest field intensity. With the Monte-Carlo simulations, we compute the mean, the standard deviation and the error rate of the order estimation.

Let $N$ be the total number of noise samples, $l_{q}$ the locally estimated OAM order at the $q$-th sample. The estimated mean is

$$
l_{\text {mean }}=\frac{1}{N} \sum_{q=1}^{q=N} l_{q} .
$$

Similarly the estimated standard deviation can be written as

$$
\sigma_{l}=\sqrt{\sum_{q=1}^{q=N} \frac{1}{N}\left(l_{q}-l_{\text {mean }}\right)^{2}} .
$$

We consider that an error arises in the estimation of the OAM order when the absolute value of the difference between the locally estimated and the actual OAM order is greater than 0.5 because this value corresponds to half of the difference between two consecutive orders.

$$
\text { ProbaError }_{l}=\frac{N_{\text {error }}}{N},
$$

where $N_{\text {error }}$ is the number of samples such that $\left|l_{q}-l\right|>0.5$. In this analysis, the error rate is the key result, since it is the equivalent to the BER (Bit Error Rate) in telecommunications. In this section, we display in Fig. 8 the evolution of the estimated error rate with the number of samples. One can see that mostly after 500 noise samples, the error rate of the local OAM order estimation has stabilized. So we set $N$ to 1000 for the next simulations.

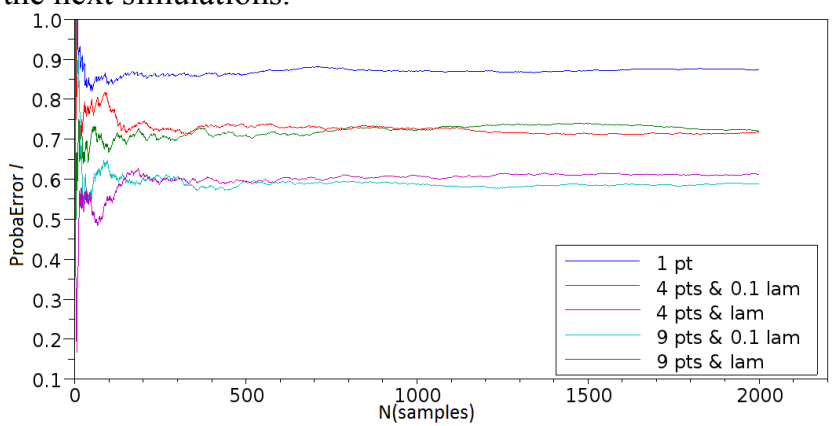

Fig. 8. Error rate for $N$ noise samples of the OAM order locally estimated. $W_{0}$ $=2 \lambda, l=2, D_{z}=10 z_{0}$ and $S N R=30 \mathrm{~dB}$.

\section{B. Results}

From the reference configuration, we study the influence of changes in one parameter $(z, l$ or $S N R)$. First and foremost, we study the sensitivity when the distance $z$ varies from 0 to 100 $z_{0}$. The error rate, in Fig. 5, confirms our previous observations. Indeed, the local estimation of $l$ seems to be impossible in the far-field zone. Increasing the number of 
elements in the VA array improves the OAM order local estimation.

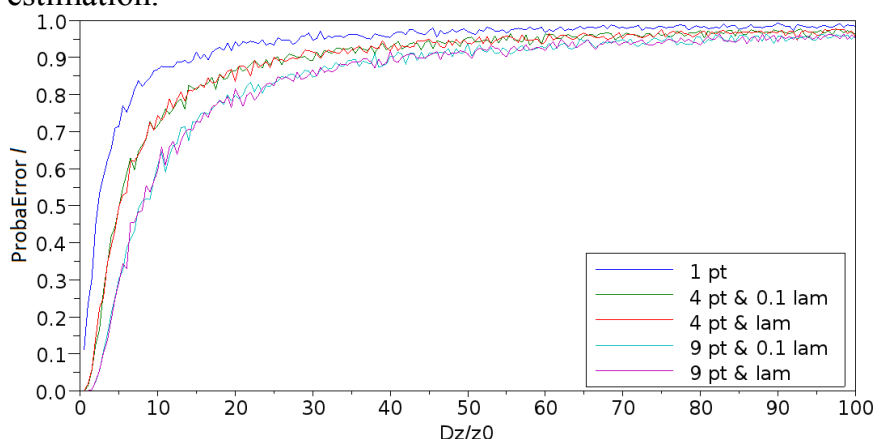

Fig. 9. Influence of $z$ on the error rate for 1000 noise samples with $W_{0}=2 \lambda$, $l=2$, and $S N R=30 \mathrm{~dB}$.

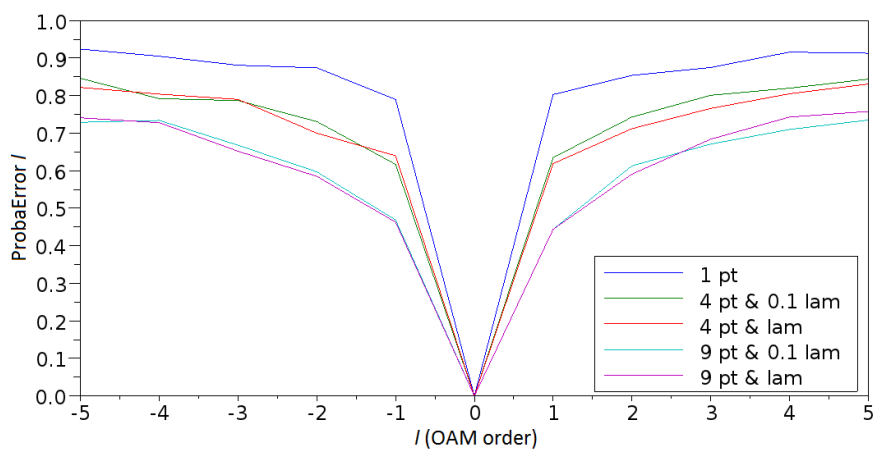

Fig. 10. Influence of $l$ on the error ratio in 1000 noise samples. $W_{0}=2 \lambda$, $z=10 z_{0}$, and $S N R=30 \mathrm{~dB}$.

Fig. 10 represents the error rate when modifying the OAM order $l$. The lower $|l|$ is, the better the estimation is. This is true regardless of the array topology. Note also that when the number of elements in the array increases, the error rate decreases.

The impact of the SNR on the OAM order estimation is given in Table. 3. As expected, the error grows rapidly when the SNR decreases.

TABLE III. INFLUENCE OF THE SNR ON THE ERROR RATE FOR 1000 NOISE SAMPLES WITH $z=10 z_{0}, W_{0}=2 \lambda, l=2$.

\begin{tabular}{|c|c|c|}
\hline SNR(dB) & 10 & 30 \\
\hline 1 VA & 0.99 & 0.85 \\
\hline 4 VA & 0.97 & 0.70 \\
\hline 9 VA & 0.95 & 0.60 \\
\hline
\end{tabular}

\section{CONCLUSION}

In this paper, we have investigated the sensitivity to noise of the local estimation of the OAM order. Several topologies of receivers have been considered consisting in either a single or an array of vector antennas that measure the 6 components of the electromagnetic fields.

The local estimation of the OAM order has been found to be very sensitive to noise. In the far-field zone and for realistic values of SNR, we have obtained error rates that are not acceptable in the context of wireless transmissions. This questions whether OAM can be directly used in this context, i.e. without dedicated signal processing algorithm. However, at shorter distances, the error rate decreases, which may lead to practical applications.

\section{REFERENCES}

[1] J.H. Poynting, "The wave motion of a revolving shaft, and a suggestion as to the angular momentum in a beam of circularly polarised light," Proc. R. Soc. A. vol. 82, no. 557, pp. 560-567,1909.

[2] R. A. Beth., "Mechanical detection and measurement of the angular momentum of light," Phys. Rev., vol. 50, pp. 115-125, 1936.

[3] L. Allen, M.W. Beijersbergen, R.J.C Spreeuw and J.P. Woerdman, "Orbital angular momentum of light and the transformation of LaguerreGaussian laser modes," Phys. Rev. A, vol. 45, pp. 8185-8189, 1992.

[4] B. Thidé et al, "Utilization of photon orbital angular momentum in the low-frequency radio domain," Phys. Rev. Lett, vol. 99, pp. 087701, 2007

[5] F. Tamburini, E. Mari, A. Sponselli, B. Thidé, A. Bianchini and F. Romanato, "Encoding many channels on the same frequency through radio vorticity: first experimental test," New Journal of Physics, vol.14, no. 3, pp 03301, 2012.

[6] O. Edfors and A. Johansson, "Is orbital angular momentum (oam) based radio communication an unexploited area?", IEEE Trans. Antennas Propag., vol. 60, no. 2, pp. 1126-1131, 2012.

[7] S.M. Mohammadi, L.K.S Daldorff, J.E.S. Bergman, R. L. Karlsson, B. Thidé, K. Forozesh, T.D. Carozzi, and B. Isham, "Orbital angular momentum in radio - A system study," IEEE Trans. Antennas Propag., vol. 5, no. 2, pp. 565-572, 2010.

[8] J. Humblet, "Sur le moment d'impulsion d'une onde électromagnétique", Physica, volume 10, no. 7, pp. 585-603, 1943.

[9] G.F. Hatke, "Conditions for unambiguous source location using polarization diverse arrays," ASILOMAR Conference, pp.1365-1369, 1993.

[10] J.P. Torres, L. Tomer, "Twisted Photons," Wiley, 2011. 